\author{
S. Fourlanos - F. Dotta - C. J. Greenbaum • \\ J. P. Palmer • O. Rolandsson - P. G. Colman • \\ L. C. Harrison
}

\title{
Latent autoimmune diabetes in adults (LADA) should be less latent
}

Received: 4 May 2005 / Accepted: 11 May 2005 / Published online: 29 September 2005

(C) Springer-Verlag 2005

\begin{abstract}
Latent autoimmune diabetes in adults' (LADA) is the term coined to describe adults who have a slowly progressive form of autoimmune or type 1 diabetes that can be treated initially without insulin injections. The diagnosis of LADA is currently based on three clinical criteria: (1) adult age at onset of diabetes; (2) the presence of circulating islet autoantibodies, which distinguishes LADA
\end{abstract}

A perspective commissioned by the Immunology of Diabetes Society and dedicated to the memory of Umberto di Mario.

S. Fourlanos · L. C. Harrison $(\bowtie)$

Autoimmunity and Transplantation Division,

The Walter and Eliza Hall Institute of Medical Research,

$1 \mathrm{G}$ Royal Parade,

Parkville, Victoria, 3050, Australia

e-mail: harrison@wehi.edu.au

Tel.: +61-3-93452468

Fax: $+61-3-93470852$

F. Dotta

Diabetes Unit, Department of Internal Medicine

and Department of Endocrine and Metabolic Sciences,

University of Siena; Policlinico Le Scotte,

Viale Bracci 18,

53100 Siena, Italy

C. J. Greenbaum

Benaroya Research Institute at Virginia Mason,

Seattle, WA, USA

\section{J. P. Palmer}

Department of Medicine, Division of Metabolism, Endocrinology and Nutrition,

Veterans Affairs Puget Sound Health Care System,

University of Washington,

Seattle, WA, USA

O. Rolandsson

Family Medicine, Public Health and Clinical Medicine,

Umeå University,

Umeå, Sweden

P. G. Colman

Department of Diabetes and Endocrinology,

The Royal Melbourne Hospital,

Parkville, Victoria, Australia from type 2 diabetes; and (3) insulin independence at diagnosis, which distinguishes LADA from classic type 1 diabetes. The prevalence of LADA in adults presenting with non-insulin-requiring diabetes is approximately $10 \%$. Recognition of LADA expands the concept and prevalence of autoimmune diabetes, but LADA remains poorly understood at both a clinical and research level. In this perspective, we review the nomenclature, diagnostic criteria, genetics, pathology and therapy of LADA, to arrive at recommendations that might advance knowledge and management of this form of diabetes.

Keywords Autoimmune diabetes - Classification · Diagnostic criteria - HLA - Latent autoimmune diabetes in adults $\cdot$ LADA . Type 1 diabetes

Abbreviations IAA: insulin autoantibodies - IA2: tyrosine phosphatase-like insulinoma antigen 2 - ICA: islet cell antibody - LADA: latent autoimmune diabetes in adults - TPO: thyroid peroxidase - TG: thyroglobulin UKPDS: UK Prospective Diabetes Study · VNTR: variable number of tandem repeats

\section{Introduction}

The term 'latent autoimmune diabetes in adults', abbreviated to LADA, was coined by Tuomi et al. [1] to describe patients with a slowly progressive form of autoimmune or type 1 diabetes who could be treated initially without insulin injections. This clinical observation was intended to distinguish LADA from classic type 1 diabetes, where insulin is required from diagnosis, and from type 2 diabetes, where insulin is not required at all or at least until some years after diagnosis. Individuals with slowly progressive type 1 diabetes had previously been identified by an immunofluorescence staining pattern of islet cell antibodies (ICAs) 'restricted' to beta cells [2, 3]. However, the emergence of LADA followed the introduction of a robust biochemical assay for antibodies to GAD [4]. In the UK Prospective Diabetes Study (UKPDS), about 10\% of adults 
with presumed type 2 diabetes at diagnosis had evidence of islet autoimmunity in the form of circulating ICA or GAD antibodies, and the majority progressed to insulin dependence within 6 years [5]. LADA is an important form of diabetes, which should be recognised, understood and managed appropriately in clinical practice, yet, in many ways, it remains an enigma. In this perspective we will examine what we think we know about LADA in order to formulate what we need to know.

\section{Nomenclature}

At the outset, it is appropriate to ask if the term LADA is still an accurate descriptor. 'Latent' in medical terminology is usually used to describe a dormant or hidden stage of a pathological process. An individual with 'latent autoimmune diabetes', by definition, should therefore have dormant or hidden autoimmune pathology, and no clinical manifestations of disease. However, this is not the case. LADA is defined by serological evidence of islet autoimmunity in the setting of reduced and declining insulin secretion. Evidence for autoimmune pathology is not latent and is a requirement for the diagnosis. Furthermore, adults are not uniquely affected. Children can also have slowly progressive or subacute autoimmune diabetes, termed 'latent autoimmune diabetes in the young' or 'LADY', which is managed with diet and oral hypoglycaemic agents for months to years before insulin is required [6].

Several alternatives to the term LADA have been proposed but also appear to be inaccurate. 'Type 1.5 diabetes' $[7,8]$ implies that individuals always have clinical features of both type 1 diabetes (insulin deficiency and islet antibodies) and type 2 diabetes (insulin resistance and obesity), but patients with LADA are not necessarily insulin resistant or obese. 'Non-insulin-requiring autoimmune diabetes' [9] does not seem appropriate given that the majority of patients become insulin requiring. 'Slowly progressive insulin-dependent or type 1 diabetes' does not meet the criterion that patients are insulin independent at diagnosis. Moreover, it is arguable whether progression to insulin dependence after diagnosis is slow, given that insulin treatment may be required within months. A reason for adopting the term LADA was that it denoted a different clinical course from 'adult-onset type 1 diabetes'. The distinction between LADA and adult-onset type 1 diabetes is based on the requirement for exogenous insulin at diagnosis, but this depends on when, how and by whom the diagnosis is made. We suggest that this distinction is artificial and, although somewhat clinically useful, does not aid the understanding of autoimmune diabetes in adults. It is conceivable that LADA and adult-onset type 1 diabetes have the same pathogenesis and are, in fact, one and the same condition, as suggested by similar HLA gene and autoantibody marker profiles [10] and by the occurrence of both LADA and adult-onset cases in extended families [11].

The term LADA served the important purpose of drawing attention to autoimmune diabetes in adults. However, in our experience, while clinicians and patients under- stand autoimmune diabetes, they are confused by the word 'latent'. We suggest that the broader term 'autoimmune diabetes' is more appropriate for use in clinical practice and that the term LADA be used in research studies until there is general agreement about whether LADA and adult-onset type 1 diabetes are the same.

\section{Diagnostic criteria}

The diagnosis of LADA is currently based on three criteria: (1) adult age at onset of diabetes; (2) the presence of circulating islet autoantibodies; and (3) lack of a requirement for insulin for at least 6 months after diagnosis. Islet autoantibodies are markers of beta cell autoimmunity that distinguish LADA from type 2 diabetes. A period of insulin independence after diagnosis is meant to distinguish LADA from classic type 1 diabetes.

\section{Age}

The minimum age cut-off for LADA varies from 25 to 40 years $[5,10,12]$, but is arbitrary. Ideally, an age cut-off would be based on longitudinal studies of the pre-clinical natural history of LADA, using the metabolic and immunogenetic markers that distinguish LADA from classic juvenile-onset type 1 diabetes. In the meantime, we would propose that the operational minimal age be 30 years.

\section{Islet autoantibodies}

The diagnosis of LADA has relied mainly on seropositivity for antibodies to GAD; insulin autoantibodies (IAA) and antibodies to tyrosine phosphatase-like insulinoma antigen 2 (IA2) were reported to be infrequent in LADA $[13,14]$. However, an accurate profile of humoral immunity in LADA requires further studies to document the prevalence of IAA, IA2 antibodies and ICA in LADA in different populations. GAD antibodies are not a sine qua non-adults with diabetes who are not insulin-dependent at diagnosis and have any islet autoantibody would currently be classified as having LADA.

\section{Insulin independence}

The other criterion for LADA is a minimum period of 6 months of insulin independence after diagnosis $[5,10,12]$. At least three factors influence the period of insulin independence: (1) the natural history of the disease; (2) the timing of diagnosis in relation to natural history; and (3) the therapeutic bias of the treating physician. These will vary from patient to patient. Asymptomatic individuals diagnosed with diabetes on the basis of raised blood glucose alone are more likely to meet the criterion of insulin independence for a minimum period than those diagnosed with diabetes after becoming symptomatic. The current 
diagnostic classification is therefore biased, often excluding patients who are symptomatic and/or have a delayed diagnosis of diabetes. Someone with asymptomatic undiagnosed diabetes for many months who eventually presents with symptoms is likely to be immediately commenced on insulin injections and, thus, considered to have classic type 1 diabetes. If diagnosed with diabetes earlier on the basis of blood glucose alone, this person would be insulin independent initially and, if islet antibody-positive, likely to be classified as having LADA, not classic type 1 diabetes. Moreover, the decision to treat LADA patients with oral agents or insulin reflects also the judgement of the treating physician. Some patients with marked insulin deficiency will be treated with oral hypoglycaemic agents when they should have been treated with insulin from the outset. Conversely, some patients with adequate endogenous insulin production will be treated with insulin from the outset when they could have achieved adequate glycaemic control with oral agents. Thus, an overriding factor is whether or not the treating physician is proactive with regard to insulin treatment. Clearly, further knowledge of the natural history and pathogenesis of LADA is required to refine objective diagnostic criteria.

Prior to the introduction of islet autoantibody assays, studies had shown that the level of C-peptide secretion helped to predict insulin requirement in adults presenting with diabetes [15]. We suggest that insulin independence in clinical practice be corroborated by formally demonstrating adequate insulin production at diagnosis. Endogenous insulin or C-peptide can be measured under fasting conditions and after stimulation with oral glucose, a mixed meal, intravenous glucagon or other secretagogues such as intravenous arginine. However, the comparative sensitivity of these stimuli, and reference ranges from the healthy population standardised for age and anthropometric indices, still need to be established. The aim should be to demonstrate satisfactory endogenous insulin production before continuing with oral hypoglycaemic agents and lifestyle modification, to achieve satisfactory glycaemic control $\left(\mathrm{HbA}_{1} \mathrm{c}<7 \%\right)$. Patients should be informed that oral hypoglycaemic agents may only be effective in the shorter term and that there is a high probability of becoming insulin dependent. Future studies should determine if early treatment with insulin preserves residual beta cell function and reduces complications in adults with autoimmune diabetes.

\section{BMI}

A clinical view that persists is that LADA patients are usually lean at diagnosis $[9,16]$, similar to children presenting with type 1 diabetes. However, documentation of the BMI in LADA populations of European extraction does not support this view. Most larger studies of LADA cohorts report a mean $\mathrm{BMI}$ in the overweight or obese categories $\left(\mathrm{BMI}>25.0 \mathrm{~kg} / \mathrm{m}^{2}\right)[6,17-19]$, and a majority report a similar BMI to type 2 diabetes cohorts $[6,18,19]$. Thus, a normal BMI $\left(<25.0 \mathrm{~kg} / \mathrm{m}^{2}\right)$ should not be a diagnostic criterion for LADA.

\section{Genes}

\section{HLA genes}

Type 1 diabetes risk is associated with genes in the MHC region (which includes the IDDM1 locus), in particular with an increased frequency of the HLA class II alleles $D R 3$ and/or $D R 4$ and $D Q 2$ and/or $D Q 8$ compared with the general population. Cross-sectional studies have shown that age at diagnosis of classic type 1 diabetes is inversely related to the frequency of highest risk HLA phenotypes [20-22].

In two large European studies on LADA $[5,13]$, the highest risk HLA phenotypes for type 1 diabetes, $D R 3 / 4$ and $D Q 2 / 8$, were more prevalent in LADA patients than in healthy control subjects, consistent with the known genetic predisposition to islet autoimmunity. However, no consistent differences in the class II alleles DR3,DR4,DQ2 or $D Q 8$ have been identified in LADA compared with classic type 1 diabetes. One study compared LADA with juvenileonset type 1 diabetes and found that the highest-risk $D Q 2 / 8$ phenotype was not as frequent in LADA [13], consistent with the later onset of diabetes [20-22]. When LADA was compared with adult-onset type 1 diabetes $[13,23]$ there were no consistent differences in class II allele frequency, suggesting that they have a similar genetic basis. Analysis of HLA class II and class I genes across the autoimmune diabetes spectrum encompassing juvenile-onset type 1 diabetes, adult-onset type 1 diabetes and LADA in different ethnic populations would further inform our understanding of the genetics of autoimmune diabetes presenting at different ages.

\section{The IDDM2 locus}

The IDDM2 genetic susceptibility locus, which maps to a variable number of tandem repeats (VNTR) upstream of INS (which encodes human proinsulin) is associated with type 1 diabetes. Short class I VNTR alleles predispose to type 1 diabetes, whereas longer class III alleles are protective [24]. It is hypothesised $[25,26]$ that IDDM2 regulates the expression of proinsulin in the thymus and thereby the selection of proinsulin-reactive $\mathrm{T}$ cells and the acquisition of immune tolerance to proinsulin. Evidence of autoimmunity to (pro)insulin in the form of autoantibodies is not a feature of LADA. Nevertheless, it may be informative to determine the frequency of IDDM2 alleles in LADA relative to the general population and to type 1 diabetes. Two studies have, in fact, implicated IDDM2 in LADA. The class I VNTR allele was more frequent in adults with diabetes who were GAD antibody-positive [27]. Compared with non-diabetic control subjects, the class I VNTR was, as in type 1 diabetes subjects, more 
frequent in LADA subjects, and the shortest class I allele was also more frequent in these individuals [28]. These findings raise the possibility that LADA subjects might be distinguished by increased $\mathrm{T}$ cell responses to proinsulin.

\section{Other genes}

More recently, the gene for MHC class I chain-related A $(M I C A)$ has been associated with both LADA and type 1 diabetes [29]. Other candidate genes in LADA include those for cytotoxic $\mathrm{T}$ lymphocyte-associated protein 4 [12] and TNF-2 [30].

\section{Pathogenesis}

The natural history of pre-clinical LADA is unknown. Consider the two extreme possibilities: patients with LADA could have either long-standing islet autoimmunity and slowly progressive beta cell damage over many years, or the onset of islet autoimmunity for the first time in adult life with a shorter pre-clinical phase. The more logical notion that LADA begins many years before diagnosis remains to be proved. Longitudinal studies of individuals with islet autoantibodies in type 1 diabetes families and in the general population, which are underway on several continents, should eventually establish whether there is a link between islet autoimmunity in childhood/adolescence and the subsequent development of LADA. The offspring of LADA patients were found to have reduced insulin secretion but no difference in insulin sensitivity compared with control subjects [31], but the interpretation of this finding is limited because a majority of the offspring were not islet autoantibody-positive.

A key observation in the UKPDS was progression to insulin dependence after diagnosis of LADA. A majority of patients with GAD antibodies at diagnosis developed insulin dependence within 6 years [5], which implies progressive autoimmune beta cell destruction. The rate of progression of the autoimmune process in LADA would be predicted to be less aggressive than in classic type 1 diabetes, because the known islet antibody specificities are mostly restricted to GAD in LADA and the number of specificities is known to predict rate of progression to clinical type 1 diabetes [32-34]. A Japanese study found that GAD antibodies in adults with 'slowly progressive type 1 diabetes' recognised unique, linear N-terminal epitopes of GAD65, and that the level of these antibodies was inversely correlated with the time to insulin dependence [35].

Information on $\mathrm{T}$ cell responses to islet autoantigens in LADA is sparse. Peripheral blood mononuclear cells from islet antibody-positive patients with phenotypic type 2 diabetes were found to react to lysed human pancreatic islets [36], but the target antigen(s) was not identified. Determining if LADA is characterised by T cells that react to specific islet antigens or epitopes in a quantitively or qualitatively distinct way is a high priority, but will require improvements in current T cell assay methodology. Studies of pancreatic histology in LADA are limited to a single case report in a 65-year-old Japanese woman, which revealed T cell infiltration of islets (insulitis) and significant residual beta cell mass [37]. Studies of pancreatic histology in LADA, on intraoperative biopsies as well as cadaveric tissue, if ethically acceptable, would greatly enhance our understanding of this condition.

\section{Autoimmune disease associations}

The association of other autoimmune diseases with type 1 diabetes, especially autoimmune thyroid disease [38] and coeliac disease [39], is well established. The clustering of these autoimmune diseases is related to common HLA DR3-DQ2, DR4-DQ8 risk haplotypes [38]. Before LADA was formally defined, a slowly progressive form of type 1 diabetes was associated with other organ-specific autoimmune diseases and seen as part of the autoimmune polyendocrine syndrome type 2 [40].

In children with type 1 diabetes, the reported prevalence of autoimmune thyroiditis is 4\% [41] and that of associated thyroid antibodies (thyroid peroxidase [TPO] antibodies, thyroglobulin [TG] antibodies) 16\% [42]. Approximately $25 \%$ of adults with autoimmune diabetes have serological evidence of autoimmune thyroiditis. A large Italian study found a high frequency of TPO antibodies in LADA patients $(24 \%)$ compared with type 2 diabetes patients $(5 \%)$ [43]. There are no reports on the frequency of clinical thyroid disease in LADA.

Coeliac disease occurs in approximately $10 \%$ of children with type 1 diabetes. A high frequency (19\%) of antibodies to gliadin was reported in LADA [44], but the prevalence of coeliac disease confirmed by biopsy is unknown. An increased frequency of antibodies to 21-hydroxylase and 17-hydroxylase, markers of autoimmune hypoadrenalism (Addison's disease), has also been reported in LADA [43].

\section{Metabolic characterisation}

Gottsäter et al. [45] found that the level of insulin secretion in LADA was intermediate between type 1 diabetes and type 2 diabetes. This key study showed that fasting and stimulated C-peptide were reduced in LADA compared with type 2 diabetes and healthy control subjects, and that a rapid decline in stimulated C-peptide secretion occurred within a few years of diagnosis in LADA patients treated with oral hypoglycaemic agents. Subsequently, several small longitudinal studies have also found a relatively rapid decline in insulin secretion in the first few years after diagnosis [46-48]. Combining the results of these four studies reveals a $50 \%$ loss of insulin secretory function over $3-$ 4 years. This rate may not accord with the prevailing view that loss of beta cell function is slowly progressive in LADA compared with type 1 diabetes. As mentioned earlier, different means of assessing residual beta cell func- 
tion by stimulating insulin or C-peptide secretion with intravenous glucose, intravenous glucagon, a mixed meal or oral glucose have not been compared.

Small studies using the homeostasis model assessment (HOMA) $[18,49]$ suggest that LADA patients are insulin resistant. However, insulin resistance has received relatively little attention in LADA and the measurement of insulin action by the benchmark hyperinsulinaemic-euglycaemic clamp technique has not been reported.

\section{Complications}

The frequency of ketoacidosis has not been documented in LADA, but is assumed to be very low. Chronic vascular complications associated with type 1 diabetes and type 2 diabetes are also present in LADA [50].

\section{Microvascular}

The frequency of retinopathy and nephropathy in LADA is similar to that in type 2 diabetes; neuropathy appears to be uncommon initially but increases with duration of disease [51]. The frequency of retinopathy (51\%), nephropathy (microalbuminuria) (27\%) and neuropathy (29\%) was similar in LADA patients and patients with type 1 diabetes diagnosed for more than 10 years; type 1 diabetic patients had significantly more retinopathy $(76 \%)$ but less neuropathy (13\%) [50]. Microvascular complications in all forms of diabetes are thought to be related to the degree of hyperglycaemia. Glycaemic control was similar in LADA and type 2 diabetes [50], perhaps accounting for the similar frequencies of microvascular complications. The lower frequency of retinopathy in LADA and type 2 diabetes compared with type 1 diabetes could be related to the older age of onset or to preservation of more beta cell function, which is known to be associated with lower rates of retinopathy [52].

\section{Macrovascular}

Macrovascular complication rates were reported to be similar in long-standing LADA and type 2 diabetes, but far lower in type 1 diabetes of similar duration [50]. However, the type 1 diabetic patients were younger, and age is likely to be an important variable. The independent associations of hypertension, hyperlipidaemia, obesity and hyperglycaemia with macrovascular disease in diabetic patients are well established. It is interesting that hypertension, hyperlipidaemia and obesity were less common in LADA than in type 2 diabetes [50], yet the rates of macrovascular complications were similar. Possible explanations include differences in pathogenesis or treatment. Given the autoimmune pathology, LADA patients may have greater systemic inflammation, implicated in vascular pathology [53]. LADA patients might also be suboptimally treated because they often start treatment with insulin later than is clinically indicated, due to unrecognised insulin deficiency and a reluctance to change from oral therapies to injections. They are also likely to have a shorter duration of treatment with metformin, an oral agent associated with a lower rate of ischaemic heart disease in the UKPDS [54]

\section{Therapy}

Any therapy in diabetes should aim for optimal glycaemic control and preservation of beta cell function, as well as being safe and practical for everyday use. Optimising glycaemic control decreases the frequency of microvascular complications in type 1 diabetes and type 2 diabetes [55]. Preservation of beta cell function is associated with im-

Table 1 Recommendations for research on LADA

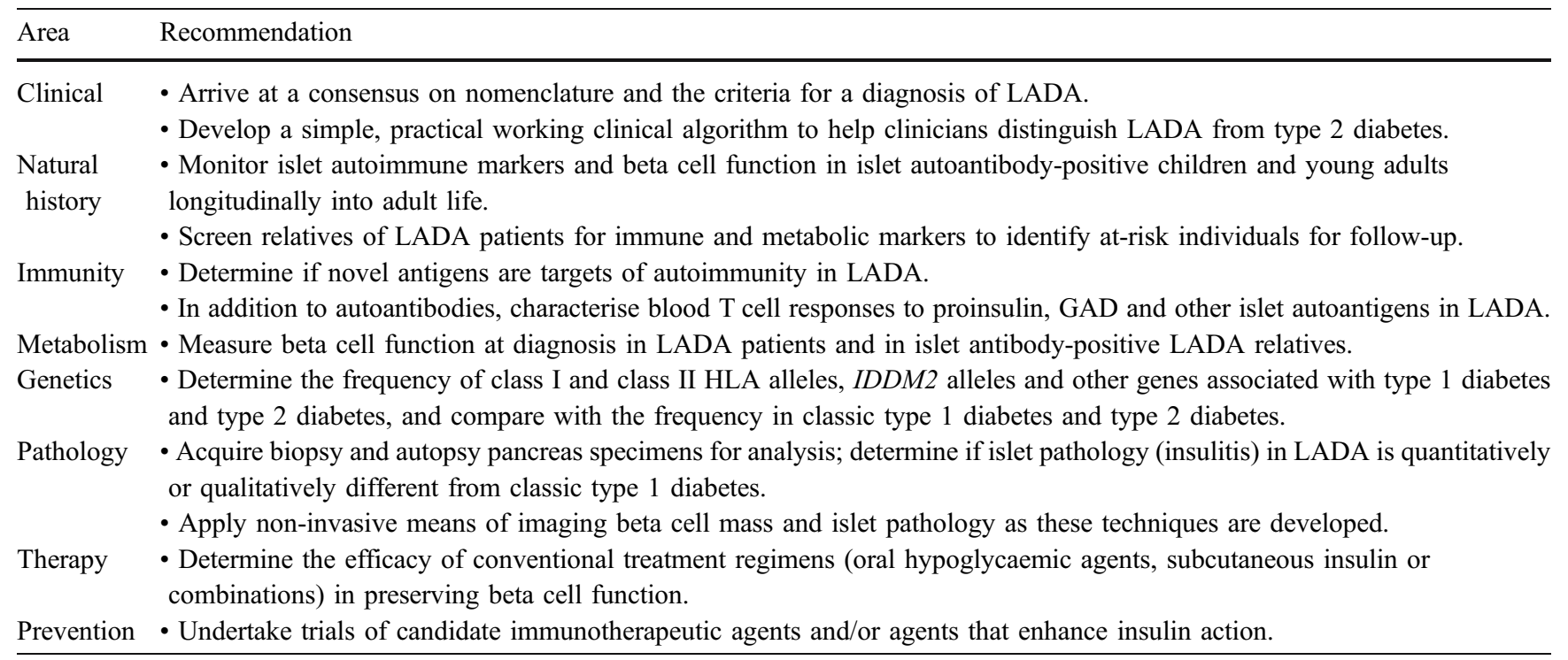


proved glycaemic control and a lower frequency of complications in type 1 diabetes [52]. The efficacy of insulin treatment after diagnosis of LADA is unknown but is currently being investigated in a large-scale Japanese study [56]. The efficacy of thiazolidinedione drugs in LADA has not been established.

Oral hypoglycaemic agents are efficacious in LADA, but it appears they do not preserve beta cell function, since the majority of patients become insulin-dependent within 6 years of diagnosis [54]. However, randomised controlled trials have not been performed to formally confirm this conclusion. A key question is the relative efficacy of each of the oral hypoglycaemic agents (sulfonylureas, biguanides, thiazolidinediones) and insulin regimens (premixed insulin twice daily, basal bolus insulin regimen), as well as their combinations, in preserving beta cell function. Emphasising preservation of beta cell function as the rationale, a consensus is required on a simple, reliable and standardised test to measure beta cell function in daily clinical practice. Although correlated with stimulated C-peptide, neither fasting C-peptide nor insulin serve as adequate markers of beta cell function as they may also reflect insulin action.

Lifestyle intervention with diet and exercise improves glycaemic control in type 1 diabetes and type 2 diabetes. Two studies, the Finnish Diabetes Prevention Study [57] and the Diabetes Prevention Program Study [58] reported that lifestyle intervention improved beta cell function in people with impaired glucose tolerance. Lifestyle intervention would presumably improve short-term glycaemic control in LADA, but studies are required to determine if it preserves beta cell function in the long term. Preventative strategies for type 1 diabetes including immune-based therapies may also be applicable to LADA. If beta cell destruction is slowly progressive in LADA, this implies a wider therapeutic window for prevention.

\section{Recommendations for an improved understanding of LADA}

LADA represents a significant component of autoimmune diabetes but, compared with classic type 1 diabetes, is poorly researched. This review of the state of our knowledge about LADA leads us to the recommendations in Table 1 for improving the understanding and management of this condition.

Acknowledgements The authors would like to thank C. McLean for secretarial assistance and her administrative support to the Immunology of Diabetes Society.

\section{References}

1. Tuomi T, Groop LC, Zimmet PZ, Rowley MJ, Knowles W, Mackay IR (1993) Antibodies to glutamic acid decarboxylase reveal latent autoimmune diabetes mellitus in adults with a noninsulin-dependent onset of disease. Diabetes 42:359-362
2. Genovese S, Bonifacio E, McNally JM et al (1992) Distinct cytoplasmic islet cell antibodies with different risks for type 1 (insulin-dependent) diabetes mellitus. Diabetologia 35:385-388

3. Gianani R, Pugliese A, Bonner-Weir S et al (1992) Prognostically significant heterogeneity of cytoplasmic islet cell antibodies in relatives of patients with type I diabetes. Diabetes 41:347-353

4. Hagopian WA, Karlsen AE, Gottsater A et al (1993) Quantitative assay using recombinant human islet glutamic acid decarboxylase (GAD65) shows that $64 \mathrm{~K}$ autoantibody positivity at onset predicts diabetes type. J Clin Invest 91:368-374

5. Turner R, Stratton I, Horton V et al (1997) UKPDS 25: autoantibodies to islet-cell cytoplasm and glutamic acid decarboxylase for prediction of insulin requirement in type 2 diabetes. UK Prospective Diabetes Study Group. Lancet 350: $1288-1293$

6. Lohmann T, Nietzschmann U, Kiess W (2000) 'Lady-like': is there a latent autoimmune diabetes in the young? Diabetes Care 23:1707-1708

7. Juneja R, Palmer JP (1999) Type 1 1/2 diabetes: myth or reality? Autoimmunity 29:65-83

8. Palmer JP, Hirsch IB (2003) What's in a name: latent autoimmune diabetes of adults, type 1.5, adult-onset, and type 1 diabetes. Diabetes Care 26:536-538

9. Pozzilli P, Di Mario U (2001) Autoimmune diabetes not requiring insulin at diagnosis (latent autoimmune diabetes of the adult): definition, characterization, and potential prevention. Diabetes Care 24:1460-1467

10. Hosszufalusi N, Vatay A, Rajczy K et al (2003) Similar genetic features and different islet cell autoantibody pattern of latent autoimmune diabetes in adults (LADA) compared with adultonset type 1 diabetes with rapid progression. Diabetes Care 26:452-457

11. Douek IF, Gillespie KM, Bingley PJ, Gale EA (2002) Diabetes in the parents of children with Type I diabetes. Diabetologia 45:495-501

12. Cosentino A, Gambelunghe G, Tortoioli C, Falorni A (2002) CTLA-4 gene polymorphism contributes to the genetic risk for latent autoimmune diabetes in adults. Ann N Y Acad Sci 958:337-440

13. Tuomi T, Carlsson A, Li H et al (1999) Clinical and genetic characteristics of type 2 diabetes with and without GAD antibodies. Diabetes 48:150-157

14. Takeda H, Kawasaki E, Shimizu I et al (2002) Clinical, autoimmune, and genetic characteristics of adult-onset diabetic patients with GAD autoantibodies in Japan (Ehime Study). Diabetes Care 25:995-1001

15. Gjessing HJ, Matzen LE, Pedersen PC, Faber OK, Froland A (1998) Insulin requirement in non-insulin-dependent diabetes mellitus: relation to simple tests of islet B-cell function and insulin sensitivity. Diabet Med 5:328-332

16. Leslie RD, Pozzilli P (1994) Type I diabetes masquerading as type II diabetes. Possible implications for prevention and treatment. Diabetes Care 17:1214-1219

17. Davis TM, Zimmet P, Davis WA, Bruce DG, Fida S, Mackay IR (2000) Autoantibodies to glutamic acid decarboxylase in diabetic patients from a multi-ethnic Australian community: the Fremantle Diabetes Study. Diabet Med 17:667-674

18. Carlsson A, Sundkvist G, Groop L, Tuomi T (2000) Insulin and glucagon secretion in patients with slowly progressing autoimmune diabetes (LADA). J Clin Endocrinol Metab 85:76-80

19. Zinman B, Kahn SE, Haffner SM, O’Neill MC, Heise MA, Freed MI (2004) Phenotypic characteristics of GAD antibodypositive recently diagnosed patients with type 2 diabetes in North America and Europe. Diabetes 53:3193-3200

20. Karjalainen J, Salmela P, Ilonen J, Surcel HM, Knip M (1989) A comparison of childhood and adult type I diabetes mellitus. N Engl J Med 320:881-886

21. Caillat-Zucman S, Garchon HJ, Timsit J et al (1992) Agedependent HLA genetic heterogeneity of type 1 insulin-dependent diabetes mellitus. J Clin Invest 90:2242-2250 
22. Tait BD, Harrison LC, Drummond BP, Stewart V, Varney MD, Honeyman MC (1995) HLA antigens and age at diagnosis of insulin-dependent diabetes mellitus. Hum Immunol 42:116-122

23. Cerna M, Novota P, Kolostova K et al (2003) HLA in Czech adult patients with autoimmune diabetes mellitus: comparison with Czech children with type 1 diabetes and patients with type 2 diabetes. Eur J Immunogenet 30:401-407

24. Bennett ST, Lucassen AM, Gough SC et al (1995) Susceptibility to human type 1 diabetes at IDDM2 is determined by tandem repeat variation at the insulin gene minisatellite locus. Nat Genet 9:284-292

25. Pugliese A, Zeller M, Fernandez A Jr et al (1997) The insulin gene is transcribed in the human thymus and transcription levels correlated with allelic variation at the INS VNTRIDDM2 susceptibility locus for type 1 diabetes. Nat Genet 15:293-297

26. Vafiadis P, Bennett ST, Todd JA et al (1997) Insulin expression in human thymus is modulated by INS VNTR alleles at the IDDM2 locus. Nat Genet 15:289-292

27. Matejkova-Behanova M, Vankova M, Hill M et al (2004) Polymorphism of INS VNTR is associated with glutamic acid decarboxylase antibodies and postprandial C-peptide in patients with onset of diabetes after 35 years of age. Physiol Res 53: 187-190

28. Cerrone GE, Caputo M, Lopez AP et al (2004) Variable number of tandem repeats of the insulin gene determines susceptibility to latent autoimmune diabetes in adults. Mol Diagn 8:43-49

29. Gambelunghe G, Ghaderi M, Tortoioli C et al (2001) Two distinct MICA gene markers discriminate major autoimmune diabetes types. J Clin Endocrinol Metab 86:3754-3760

30. Vatay A, Rajczy K, Pozsonyi E et al (2002) Differences in the genetic background of latent autoimmune diabetes in adults (LADA) and type 1 diabetes mellitus. Immunol Lett 84:109115

31. Vauhkonen I, Niskanen L, Knip M et al (2000) Impaired insulin secretion in non-diabetic offspring of probands with latent autoimmune diabetes mellitus in adults. Diabetologia 43:69-78

32. Bingley PJ, Bonifacio E, Williams AJ, Genovese S, Bottazzo GF, Gale EA (1997) Prediction of IDDM in the general population: strategies based on combinations of autoantibody markers. Diabetes 46:1701-1710

33. Verge CF, Stenger D, Bonifacio E et al (1998) Combined use of autoantibodies (IA-2 autoantibody, GAD autoantibody, insulin autoantibody, cytoplasmic islet cell antibodies) in type 1 diabetes: combinatorial islet autoantibody workshop. diabetes 47 : $1857-1866$

34. Harrison LC (2001) Risk assessment, prediction and prevention of type 1 diabetes. Pediatric Diabetes 2:71-82

35. Kobayashi T, Tanaka S, Okubo M, Nakana, Murase T, Lernmark A (2003) Unique epitopes of glutamic acid decarboxylase autoantibodies in slowly progressive type 1 diabetes. J Clin Endocrinol Metab 88:4678-4775

36. Brooks-Worrell BM, Juneja R, Minokadeh A, Greenbaum CJ, Palmer JP (1999) Cellular immune responses to human islet proteins in antibody-positive type 2 diabetic patients. Diabetes 48:983-988

37. Shimada A, Imazu Y, Morinaga S et al (1999) T-cell insulitis found in anti-GAD65+diabetes with residual beta-cell function. A case report. Diabetes Care 22:615-617

38. Weetman AP (2003) Autoimmune thyroid disease: propagation and progression. Eur J Endocrinol 148:1-9

39. Smith CM, Clarke CF, Porteous LE, Elsori H, Cameron DJ (2000) Prevalence of coeliac disease and longitudinal follow-up of antigliadin antibody status in children and adolescents with type 1 diabetes mellitus. Pediatr Diabetes 1:199-203

40. Wagner R, Genovese S, Bosi E et al (1994) Slow metabolic deterioration towards diabetes in islet cell antibody positive patients with autoimmune polyendocrine disease. Diabetologia $37: 365-371$
41. Radetti G, Paganini C, Gentili L et al (1995) Frequency of Hashimoto's thyroiditis in children with type 1 diabetes mellitus. Acta Diabetol 32:121-124

42. Hansen D, Bennedbaek FN, Hansen LK, Hoier-Madsen M, Jacobsen BB, Hegedus L (1999) Thyroid function, morphology and autoimmunity in young patients with insulin-dependent diabetes mellitus. Eur J Endocrinol 140:512-518

43. Gambelunghe G, Forini F, Laureti S et al (2000) Increased risk for endocrine autoimmunity in Italian type 2 diabetic patients with GAD65 autoantibodies. Clin Endocrinol (Oxf) 52:565573

44. Kucera P, Novakova D, Behanova M, Novak J, TlaskalovaHogenova H, Andel M (2003) Gliadin, endomysial and thyroid antibodies in patients with latent autoimmune diabetes of adults (LADA). Clin Exp Immunol 133:139-143

45. Gottsäter A, Landin-Olsson M, Fernlund P, Lernmark A, Sundkvist G (1993) Beta-cell function in relation to islet cell antibodies during the first $3 \mathrm{yr}$ after clinical diagnosis of diabetes in type II diabetic patients. Diabetes Care 16:902-910

46. Kobayashi T, Nakanishi K, Murase T, Kosaka K (1996) Small doses of subcutaneous insulin as a strategy for preventing slowly progressive beta-cell failure in islet cell antibodypositive patients with clinical features of NIDDM. Diabetes 45:622-626

47. Torn C, Landin-Olsson M, Ostman J, Schersten B, Arnqvist H, Blohme $\mathrm{G}$ et al (2000) Glutamic acid decarboxylase antibodies (GADA) is the most important factor for prediction of insulin therapy within 3 years in young adult diabetic patients not classified as Type 1 diabetes on clinical grounds. Diabetes/ Metab Res Rev 16:442-447

48. Borg H, Gottsater A, Landin-Olsson M, Fernlund P, Sundkvist G (2001) High levels of antigen-specific islet antibodies predict future beta-cell failure in patients with onset of diabetes in adult age. J Clin Endocrinol Metab 86:3032-3038

49. Behme MT, Dupre J, Harris SB, Hramiak I, Mahon JL (2003) Insulin resistance in latent autoimmune diabetes of adulthood. Ann N Y Acad Sci 1005:374-377

50. Isomaa B, Almgren P, Henricsson M et al (1999) Chronic complications in patients with slowly progressing autoimmune type 1 diabetes (LADA). Diabetes Care 22:1347-1353

51. Baum P, Hermann W, Verlohren HJ, Wagner A, Lohmann T, Grahmann F (2003) Diabetic neuropathy in patients with 'latent autoimmune diabetes of the adults' (LADA) compared with patients with type 1 and type 2 diabetes. J Neurol 250:682-687

52. The Diabetes Control and Complications Trial Research Group (1998) Effect of intensive therapy on residual beta-cell function in patients with type 1 diabetes in the diabetes control and complications trial. A randomized, controlled trial. Ann Intern Med 128:517-523

53. Ridker PM (2003) High-sensitivity C-reactive protein and cardiovascular risk: rationale for screening and primary prevention. Am J Cardiol 92:17K-22K

54. UK Prospective Diabetes Study (UKPDS) Group (1998) Effect of intensive blood-glucose control with metformin on complications in overweight patients with type 2 diabetes (UKPDS 34). Lancet 352:854-865

55. UK Prospective Diabetes Study (UKPDS) Group (2002) Effect of intensive therapy on the microvascular complications of type 1 diabetes mellitus. JAMA 287:2563-2569

56. Maruyama T, Shimada A, Kanatsuka A et al (2003) Multicenter prevention trial of slowly progressive type 1 diabetes with small dose of insulin (the Tokyo study): preliminary report. Ann N Y Acad Sci 1005:362-369

57. Uusitupa M, Louheranta A, Lindstrom J et al (2000) The finnish diabetes prevention study. Br J Nutr 83(Suppl 1):S137S142

58. Knowler WC, Barrett-Connor E, Fowler SE et al (2002) Reduction in the incidence of type 2 diabetes with lifestyle intervention or metformin. N Engl J Med 346:393-403 